\title{
LA ONTOLOGÍA SOCIAL DE JOHN R. SEARLE Y DE MAURIZIO FERRARIS. NOTAS CRÍTICAS.
}

\author{
THE SOCIAL ONTOLOGY OF JOHN R. SEARLE AND OF \\ MAURIZIO FERRARIS. CRITICAL NOTES.
}

FERNÁN RIOSECO P.*

\section{Resumen}

En este trabajo presentamos una versión detallada de las teorías de John Searle y Maurizio Ferraris sobre la ontología social como ontología regional. En primer lugar, ofrecemos una crítica de la teoría intencional y representacional de Searle, mostrando sus aciertos y elementos problemáticos. A continuación, analizamos la teoría de la documentalidad de Ferraris, en especial, las nociones de huella y registro, cuya genealogía arranca de las distinciones conceptuales de Jacques Derrida en De la grammatalogie. En la conclusión, esperamos mostrar que ninguna de las teorías analizadas constituye una explicación suficiente de los problemas ontológicos, semánticos y epistemológicos que plantea la ontología social.

\section{Palabras Clave}

Ontología social; John Searle; intencionalidad colectiva; Maurizio Ferraris; documentalidad.

Artículo recibido para su evaluación el 9 de noviembre de 2021, y aprobado para su publicación el 20 de diciembre de 2021.

"Magíster en Filosofía, Máster en Argumentación Jurídica Universidad de Alicante, Doctorando en Derecho Universidad de Valparaíso; flrioseco@gmail.com; https://orcid. org/0000-0002-2272-0039. 


\begin{abstract}
In this work we present a detailed version of the theories of John Searle and Maurizio Ferraris on social ontology as regional ontology. First, we offer a critique of Searle's intentional and representational theory, showing its successes and problematic elements. Next, we analyze Ferraris' documentary theory, especially the notions of trace and register, whose genealogy starts from the conceptual distinctions of Jacques Derrida in de la grammatalogie. In conclusion, we hope to show that none of the analyzed theories constitutes a sufficient explanation of the ontological semantic and epistemological problems posed by social ontology.
\end{abstract}

\title{
Keywords
}

Social ontology; John Searle; collective intentionality; Maurizio Ferraris; documentation.

\section{INTRODUCCION.}

La ontología social es la disciplina que se ocupa del problema de la realidad social, esto es, aquella porción de la realidad que concierne al ser humano y sus instituciones, así como la clase de objetos o entidades que la conformarían y sus relaciones, tanto con el mundo físico como con el mundo social. La ontología social, como toda disciplina rigurosa, se basa en ciertos presupuestos metodológicos: (i) que, en un sentido filosófico amplio, es posible afirmar la existencia de la realidad; y (ii) que la realidad social es, por definición, una porción de la primera, de modo que entre ambas se da una relación de parte a todo. Desde esta perspectiva la ontología social se insertaría dentro de lo que Husserl llamó "ontologías regionales", esto es, dominios constituidos de conocimiento eidético, en la relación entre fenomenología y ontología. ${ }^{1}$

Ahora bien, la existencia de un mundo social plantea un enigma ontológico: ¿cómo puede hablarse de "realidad" respecto de hechos

1 De acuerdo con Husserl, la lógica se aviene con lo que él denomina ontología formal, mientras que la ontología contiene una serie de ontologías regionales. Así, una ontología regional sería la llamada "ontología de la naturaleza" en tanto ciencia eidética de la naturaleza física. Véase HUSSERL, Edmund: Ideen zu einer reinen Phänomenologie und phänomenologischen Philosophie. Die Phänomenologie und die Fundamente der Wissenschaften (Ideen III), Beilagen, § 6, Hua V, Den Haag, 1952, pp. 19-31. 
y objetos creados por la intencionalidad humana? Además, desde la perspectiva epistemológica, ¿cómo puede un producto de la intencionalidad humana incluir datos objetivos disponibles para la investigación y el descubrimiento en las ciencias sociales? ${ }^{2}$

Los principales teóricos de la ontología social defienden que ella es la base de las ciencias sociales y la filosofía política. ${ }^{3}$ La ontología jurídica, siguiendo la terminología de Husserl y, en nuestras latitudes, de Gustavo Bueno, ${ }^{4}$ sería una ontología local o regional en relación con la más vasta ontología social. Sin embargo, esta afirmación lejos de aclarar el asunto lo complica todavía más, pues obliga a elaborar una descripción, ya no un concepto, tanto de la ontología social como de la ontología jurídica.

Antes de abordar estas cuestiones es preciso señalar que la ontología social es diferente de la realidad natural. Como observa Tuzet, ${ }^{5}$ se debe distinguir entre la ontología-proceso y la ontología-resultado. Tradicionalmente los teóricos del derecho han estado más interesados en los problemas específicos de la ontología-resultado: ¿qué son las reglas jurídicas válidas? ¿cuál es su naturaleza? ¿cuándo puede decirse que pertenecen a un sistema jurídico determinado? ¿estas reglas mantienen relaciones de causalidad o dependencia con hechos o actos del mundo físico? ¿en qué se diferencian de otras reglas como las de los juegos? Sin embargo, en los últimos años ha tenido lugar un aumento sostenido de interés en torno a la temática propia de la ontología-proceso, esto es, a las condiciones bajo las cuales el derecho es generado.

Un problema diferente, asumido por la mayoría de los ontólogos sociales, es determinar si lo que llamamos "realidad" es un concepto unitario, de forma que la realidad social participaría de dicha unidad (solo que tendría ciertos rasgos específicos que la distinguirían) o si, por

2 THOMASSON, Amie: "Foundations for a Social Ontology". En: Protosociology: An International Journal of Interdisciplinary Research, vol. 18-19, 2003. pp. 269-290.

3 GILBERT, Margaret: On Social Facts, 1989, Routledge; SEARLE, John: La construcción de la realidad social. Barcelona, 1997, Paidós; SEARLE, John: Making the Social World: The Structure of Human Civilization, New York, 2010, Oxford University Press.

4 BUENO, Gustavo, Ensayos Materialistas, Taurus, Madrid, 1972. p. 59.

5 TUZET, Giovanni: Ontología social y práctica jurídica: un debate, tr. D. Dei Vecchi. Discusiones, XIV, 2014. p. 11. 
el contrario, no cabe hablar de unidad, existiendo diferentes realidades autónomas e independientes, una de las cuales sería la realidad social. ${ }^{6}$

En este trabajo sostenemos que la teoría de John Searle sobre la creación de los hechos sociales es insuficiente para abordar satisfactoriamente todos los problemas ontológicos, epistemológicos y semánticos asociados a la construcción de la realidad social. Ello es así porque los hechos y objetos sociales, al ser creados intencional y colectivamente, enfrentan dificultades similares a las objeciones enderezadas en contra de las teorías creacionistas en el ámbito de la ficción literaria y en el más vasto mundo de la ontología del arte y la cultura. Así, por ejemplo, es cierto que Conan Doyle creó a Sherlock Holmes en un instante determinado del tiempo $t$ y que el personaje mantiene con su autor una relación de dependencia, pero el enunciado "Sherlock Holmes no existe" parece intuitivamente verdadero, en al menos uno de los sentidos posibles de la palabra verdad. Si extrapolamos este problema a algunos de los objetos típicos de la ontología social, tales como el dinero, el matrimonio, las sinfonías y las leyes dictadas por el Parlamento, se afronta un escollo similar: su creación (por medio de la intencionalidad colectiva) depende decisivamente de las creencias individuales relativas al fenómeno social, con lo cual emerge el problema de la autorreferencialidad de los conceptos que designan hechos y objetos sociales. Puede que una versión débil de la tesis searleana, esto es, reducir la teoría sólo a los hechos institucionales, más no a todos los hechos sociales en general, permita superar estos escollos, pero al precio de debilitar la teoría dejando sin explicación y sin lugar a un sinnúmero de objetos cada vez más abstractos de la ontología social.

En el caso de la teoría de la documentalidad de Maurizio Ferraris, uno de los elementos problemáticos es que no queda claro cómo es que las huellas pueden crear, por ejemplo, obligaciones "reales", en lugar de meros sentimientos o creencias. Dicho de otro modo, no es evidente el procedimiento a través del cual los sujetos producen objetos sociales. ¿Es una combinación entre el acto y el objeto físico? A esta y otras interrogantes nos abocaremos en las páginas que siguen.

6 Searle es uno de los principales defensores de la primera tesis, esto es, aquella que afirma que la realidad es una sola, aunque la explicación de la realidad social se sustenta en la existencia de lo que Searle llama hechos institucionales, frente a los hechos brutos que serían explicativos de la realidad descrita por las ciencias naturales. 


\section{LA TEORÍA DE LOS HECHOS INSTITUCIONALES.}

En su libro La construcción de la realidad social, publicado en 1995, la pregunta originaria que plantea John Searle es cómo encaja la realidad social en una realidad más amplia como la descrita por la física, la química y la biología evolucionista. Esta reflexión inicial conduce a la distinción entre hechos brutos ${ }^{7} \mathrm{y}$ hechos institucionales. Los primeros existen con total independencia de los deseos, creencias y opiniones humanas, mientras que los segundos deben su existencia a un acuerdo colectivo y sólo existen en el marco de las instituciones sociales. Searle lo plantea en los siguientes términos:

Este libro está dedicado a un problema que me ha intrigado durante mucho tiempo: hay porciones del mundo real, hechos objetivos en el mundo, que son hechos solo merced al acuerdo humano [...] llamo a los hechos institucionales así porque para su existencia, requieren instituciones humanas (Searle 1995: 21).

A Searle le intriga la ontología de la realidad social y su pretensión es explicar cómo es que existen las instituciones sociales, y los hechos sociales e institucionales. El filósofo estadounidense quiere dar una respuesta adecuada a difíciles preguntas ontológicas: ¿cómo creamos una realidad social e institucional objetiva? ¿Son posibles los hechos institucionales? ¿Cuál es la naturaleza de esos hechos? Además, Searle está convencido que la ontología social es el fundamento de las ciencias sociales y de la filosofía política, de modo que una explicación adecuada de la primera incidirá directamente en la elucidación de las segundas.

De acuerdo con Searle, la realidad social es creada intencionalmente a través de la asignación colectiva de funciones para ciertos objetos o hechos, de acuerdo con reglas constitutivas. Las funciones asignadas exceden los componentes físicos y químicos del objeto. Estas tres condiciones (intencionalidad colectiva, asignación de funciones y reglas constitutivas) son necesarias y suficientes para que pueda hablarse de una realidad social e institucional. Como lo explica en su obra posterior Making of Social World: The Structure of Human Civilization,

7 La expresión "hechos brutos" fue acuñada por G. E. M. Anscombe en su ensayo "On Brute Facts”, Analysis, vol. 18, 1958; ahora en G. E. M. ANscomBE, 1981. pp. 22-25. 
[Los hechos institucionales] surgen cuando colectivamente se asigna una función a un fenómeno cuya composición física es insuficiente para garantizar su cumplimiento. ${ }^{8}$

El caso paradigmático es el dinero. Los componentes físicos y químicos del billete de dólar no difieren sustantivamente de otros pedazos de papel. Su valor no es intrínseco, sino extrínseco: está dado por la aceptación social, que Searle denomina estatus. Un fenómeno similar ocurre con la institución del matrimonio, ya que sin seres humanos no puede hablarse propiamente de matrimonio. En ambos casos, a través de la intencionalidad colectiva se asigna un estatus a un objeto o hecho bruto, y a ese estatus se le impone una función. Ésta es denominada función de estatus porque no puede ser cumplida meramente en virtud de los rasgos o componentes físicos del objeto, sino que depende de que se acepte o reconozca colectivamente el nuevo estatus.

Cuando este procedimiento se regulariza (en el sentido que se conforma a determinadas reglas) puede ser representado a través del lenguaje y, además, formalizado lógicamente: "X $\mathrm{X}$ cuenta como $\mathrm{Y}$ en el contexto $\mathrm{C}$ », donde $\mathrm{X}$ es un hecho $\mathrm{u}$ objeto previo, $\mathrm{Y}$ es el estatus y la función asignada colectivamente, y $\mathrm{C}$ es el marco o institución social. ${ }^{9}$ Searle concluye que un hecho institucional es todo hecho que posee la estructura lógica de la regla constitutiva «X cuenta como $\mathrm{Y}$ en el contexto $\mathrm{C}$ ». A su turno, una institución es cualquier sistema de reglas constitutivas que, una vez establecida, proporciona una estructura dentro de la cual se pueden crear hechos institucionales. ${ }^{10}$

Sin embargo, no debe pensarse que estas declaraciones constituyen una aprobación de la función de estatus y del hecho institucional. Estas declaraciones colectivas son sólo de aceptación o reconocimiento. No se trata de una aprobación porque, de hecho, alguien podría resistirse o no estar de acuerdo con funciones de estatus y reglas constitutivas de instituciones sociales, pero es evidente que ello no afectará la existencia misma de la institución. Un ladrón, por ejemplo, seguramente no estará de acuerdo con la institución de la propiedad privada y, menos aún, con la norma del Código Penal que castiga el delito de robo. Pero su rechazo

8 SEARLE, John: Making the Social World, op. cit., p. 8, traducción nuestra.

9 JIMENEZ CANO, Rosa: "El derecho como institución social: Searle y Hart". En: Entre la ética, la política y el derecho: estudios en homenaje al profesor Gregorio PecesBarba, tomo II: Teoría y Metodología del Derecho, Madrid, Dykinson, 2008. pp. 685-86.

10 Ídem, p. 687. 
de los hechos institucionales no acarrea la inexistencia de la institución (la propiedad y el Código Penal), sino sólo el quebrantamiento de una regla. ${ }^{11}$

\section{Intencionalidad colectiva.}

La intencionalidad es uno de los conceptos importantes en filosofía de la mente. De hecho, el propio Searle publicó en 1983 un libro al que tituló Intentonality. A essay in the Philosophy of Mind en el que expone varias de las ideas que luego utilizará en The Construction of Social Reality, y en Making of Social World: The Structure of Human Civilization. Dice Searle,

El puente que une los fenómenos físicos brutos y la sociedad está constituido por la intencionalidad colectiva, y el movimiento decisivo del tránsito de creación de la realidad social es la imposición intencional colectiva de función a entidades que no podrían cumplir la función sin esta imposición ${ }^{12}$.

En la teoría de Searle los hechos y objetos institucionales se crean en el marco de una institución, la que es previa a la génesis del hecho institucional. El punto crucial es que Searle entiende la institución en términos de reglas constitutivas. Para Searle la intencionalidad colectiva es un fenómeno primitivo e innato. Sin embargo, la intencionalidad cualquiera que ella sea (individual o colectiva) siempre está en la mente de los individuos. No hay una mente o consciencia colectiva por sobre los individuos.

La estructura de la intencionalidad (tanto la individual como la colectiva) es independiente del hecho de si los individuos tienen visiones correctas o erróneas acerca de la realidad. ${ }^{13}$ Es decir, es posible partir de un error radical. Además, las intenciones colectivas solo existen en los estados mentales de los individuos. Normalmente las "we-intentions" (nosotros intentamos hacer) son compartidas, pero no es una condición necesaria. Puede no estar presente este requisito e igualmente haber intencionalidad

11 En este sentido Mañalich considera que una de las ventajas de concebir el hecho punible como la "desautorización de una norma que es criterio de comportamiento jurídicamente correcto", es que de ese modo se vuelve reconocible su ontología como hecho institucional. Véase MAÑALICH, Juan Pablo: "Norma e imputación como categorías del hecho punible", En: Revista de Estudios de la Justicia, $\mathrm{N}^{\circ} 12,2010$. p. 172.

12 SEARLE, John, La construcción de la realidad social, op. cit., p. 58.

13 SEARLE, John: "Collective Intentions and Actions". En COHEN, P. MORGAN, J y POLLACK, M. E. (eds): Intentions in Communications. Cambridge, MA: Bradford Books, MIT Press, 1990. p. 407. 
colectiva, ya que para Searle la "we-intentions" es una forma primitiva de intencionalidad, que presupone un background (trasfondo) de sentido.

Como explica Isabel Lucena "la hipótesis del trasfondo fue introducida por Searle en el contexto de la filosofía del lenguaje en relación con la dirección de ajuste para que se diesen condiciones de satisfacción de significados literales". ${ }^{14}$ Es interesante observar que, según Searle, ni Noam Chomsky con su gramática generativa ni Jerry Fodor con su análisis del lenguaje del pensamiento (mentalés), y ni siquiera Sigmund Freud con su apelación al inconsciente, explicaron de manera adecuada el papel causal que cumplen las reglas en la esfera de los hechos institucionales.

La hipótesis del trasfondo permite explicar un problema importante: la institución se entiende en términos de reglas constitutivas. Pero ¿qué pasa si no se conocen las reglas? A este cuestionamiento Searle contesta que el trasfondo es preintencional. Lo que ocurre es que las personas se comportan conforme a las reglas de la institución no porque sigan las reglas que establece aquella, sino porque su propia estructura neurofisiológica les predispone a actuar conforme a las reglas de las instituciones. Dice Searle,

Para explicar cómo podemos relacionarnos con estructuras de desempeño de papeles como el lenguaje, la propiedad o el dinero, el matrimonio, etc., cuando no conocemos las reglas y, por tanto, no las podemos observar ni consciente ni inconscientemente, tengo que apelar a la noción $[\ldots]$ del trasfondo. ${ }^{15}$

Si hubiera que resumir en una frase la tesis searleana en torno a la intencionalidad, sería esta: la intencionalidad colectiva es el fundamento de la sociedad. La capacidad para la conducta colectiva es un fenómeno biológico primitivo, pero no es un rasgo exclusivo de los seres humanos. Además, es innata pues no necesita aparato cultural o lingüístico. El fundamento para Searle es obvio: la adaptación inclusiva es más exitosa cuando hay cooperación con los miembros de la misma especie. En este esquema los hechos institucionales son una subclase de hechos sociales. Todos los hechos sociales se explican por mediación de la intencionalidad colectiva.

14 LUCENA, Isabel: "La intencionalidad colectiva: un modelo explicativo de la acción y de la aceptación social”. En: Revista Telemática de Filosofía del Derecho, $\mathrm{N}^{\circ} 14$, 2011. p. 10.

15 SEARLE, John, La Construcción de la realidad social, op. cit. p. 40. 


\section{Autorreferencialidad.}

Una de las tesis importantes que Searle expone en La construcción de la realidad social es que los términos que designan objetos institucionales son lógicamente autorreferenciales, ya que para que algo caiga bajo un concepto ' $\mathrm{Y}$ ' (dinero) es necesaria la creencia colectiva de que un objeto o hecho ' $\mathrm{X}$ ' (billete de dólar) satisface la definición del término en un contexto 'C' (marco social). Es decir, nuestras creencias son parte constitutiva del fenómeno social. Esta función autorreferencial es esencial para distinguir a los objetos sociales de aquellos pertenecientes a una clase natural. Una molécula de agua $\left(\mathrm{H}_{2} \mathrm{O}\right)$ ha sido, es y seguirá siendo una molécula de agua, aunque nadie piense absolutamente nada sobre ella. Pero un objeto institucional sólo lo será en la medida en que satisfaga las condiciones de su creación y el término que lo designa sea lógicamente autorreferencial. Por el contrario, como observa Hilary Putnam con su famoso experimento de la Tierra Gemela, el significado de los términos de una clase natural no está en la mente de los hablantes, y la referencia difiere aunque los estados mentales o psicológicos de los hablantes sean los mismos ${ }^{16}$, algo que no ocurre en el caso de un hecho u objeto institucional, que dependen lógicamente de la idea de autorreferencialidad.

\section{La ontología de la realidad social.}

Siguiendo las ideas centrales de La construcción de la realidad social, en su libro Making the Social World: The Structure of Human Civilization Searle mantiene que hay una única realidad, evitando cuidadosamente postular la existencia de distintos reinos ontológicos (mental, físico, social, etc.). El derecho (en tanto sistema de normas o enunciados normativos compartidos por una comunidad) es una construcción mental, un objeto del pensamiento, pues sus componentes no poseen existencia física tangible. Esto no significa que Searle sea un constructivista o que piense que los sistemas jurídicos son puramente ideales o que no forman parte del mundo social. Para Searle las normas y los órdenes normativos existen como hechos institucionales. ${ }^{17} \mathrm{Su}$ interés es explicar cómo encajamos los seres humanos en esa única realidad, es decir, cómo nuestras ideas, pensamientos, creencias, sentimientos, tienen cabida en el mundo real. Una

16 PUTNAM, Hilary: The Meaning of 'Meaning'. University of Minnesota Press, Minneapolis, 1975. p. 137.

17 Sin embargo, Searle no se pronuncia en forma clara acerca del estatus ontológico de los hechos institucionales. 
de las conclusiones importantes es que el lenguaje desempeña un papel fundamental en la creación, constitución y mantenimiento de la realidad social. El lenguaje crea y mantiene las complejas estructuras de las instituciones sociales humanas. Nuestro mundo social es un mundo creado y sostenido por el lenguaje.

Ahora bien, en el mundo real social (identificable a través de coordenadas espaciotemporales) existen, de hecho, sistemas jurídicos positivos válidos que regulan la conducta humana. Pero cuando se afirma que un sistema jurídico ' $\mathrm{X}$ ' existe en algún lugar, lo que se quiere decir es que una determinada sociedad ' $\mathrm{Y}$ ' se ha procurado un cierto ordenamiento (jurídico). Tal ordenamiento no es otra cosa que un orden institucional, desde que las normas son hechos institucionales. ${ }^{18}$

Pese a que Searle no utiliza en forma explícita el término "artefacto", su teoría parece compatible con la idea de artefacto abstracto que Amie Thomasson postula como explicación de la naturaleza ontológica de los personajes de ficción literaria y de otros objetos abstractos como leyes, gobiernos y sinfonías. Creemos sin embargo que, si la teoría de los hechos institucionales es correcta, explicaría de mejor manera por qué el derecho es un artefacto abstracto de la cultura. ${ }^{19}$

\section{Crítica de la teoría de los hechos institucionales.}

Pese a ser una propuesta interesante y robusta acerca de cómo se construye la realidad social, la teoría de los hechos institucionales ha sido cuestionada en prácticamente todos sus planteamientos básicos.

4.1 El problema de la autorreferencialidad.

En su ensayo Realism and Human Kinds, Amie Thomasson cuestiona la tesis searleana de que todos los conceptos sociales son autorreferenciales, usando contraejemplos de distintas ciencias sociales

18 BENGOETXEA, Joxerramon: "Teoría institucional del derecho". En: Enciclopedia de Filosofia y Teoría del Derecho, vol. uno, J. Fabra y A. Núñez ed., UNAM, México 2015. p. 217.

19 Brian Epstein considera que el papel de la intencionalidad (individual o colectiva) en la ontología social está sobreestimado y es menos esencial de lo que ordinariamente se cree, de modo que podría prescindirse del enfoque intencional en cualquiera de sus variantes. Cfr. EPSTEIN: "Social Objects Without Intentions". En: Anita Konzelmann Ziv \& Hans Bernhard Schmid (eds.). Institutions, Emotions, and Group Agents: Contributions to Social Ontology, 2013. pp. 53-68. 
que, en general, se refieren a lo que la autora estadounidense denomina "estructuras de poder". ${ }^{20}$

De acuerdo con Thomasson, la teoría de los hechos institucionales es incapaz de explicar fenómenos económicos como la inflación o la recesión, ya que éstos existen o pueden existir sin que nadie tenga ninguna creencia al respecto. La inflación y la recesión son fenómenos autónomos explicados por la ciencia económica, cuyo estatus ontológico es similar al de otros fenómenos como los ciclos de la economía o los roles de género en una comunidad. Esta clase de entidades sociales son opacas epistémica y conceptualmente. Epistémicamente, porque existen o pueden existir incluso si nadie en absoluto cree que existen; conceptualmente, porque existen o pueden existir incluso si nadie considera que el objeto satisface de manera relevante el concepto asociado al término. En otras palabras, algo puede ser el caso, aunque nadie crea que es el caso; y algo puede ser el caso, aunque nadie piense jamás en ello.

Coincidimos con Burman ${ }^{21}$ en que la objeción de Thomasson es crucial para la teoría de los hechos institucionales. Si la teoría de Searle no puede manejarla adecuadamente, su potencial para explicar el mundo social queda severamente restringido. Burman considera posible mantener una tesis débil de autorreferencialidad para el caso de los conceptos opacos que constituyen estructuras de poder en el marco de las ciencias sociales, pero no podemos extendernos en su consideración.

4.2 Insuficiencia de la forma lógica $\mathrm{X}$ cuenta como $\mathrm{Y}$ en el contexto $\mathrm{C}$.

En una lúcida crítica, Barry $\mathrm{Smith}^{22}$ demostró que existen varios casos similares a los expuestos por Searle en La construcción de la realidad social en los que no parece funcionar la forma lógica «X cuenta como $\mathrm{Y}$ en el contexto C». Los contraejemplos de Smith son interesantes porque tienen que ver con el derecho y, específicamente, con algunas ficciones jurídicas:

Searle podría argüir que mi derecho de propiedad en relación con, digamos una parcela de tierra determinada es explicado de la siguiente

20 THOMASSON, Amie: "Realism and Human Kinds". En: Philosophy and Phenomenological Research, 67, 2003. pp. 580-601.

21 BURMAN, Asa: "Extending the scope of Searle's Theory of Social Reality - How to account for opaque social phenomena like power structures". En: ProtoSociology, 2015. pp. 1-18.

22 SMITH, Barry: “John Searle: From speech acts to social reality". En: B. Smith (ed.), John Searle, Cambridge, Cambridge University Press, 2003. pp. 1-33. 
manera: hay un cierto elemento físico, las escrituras de propiedad en mi caja fuerte, que cuenta como el derecho de propiedad en ciertos contextos. Una vez más, sin embargo, parece que las escrituras sólo graban o registran la existencia del derecho de propiedad. Una nota [que diga "te debo"], de manera similar, registra la existencia de una deuda, pero no cuenta como la deuda. Más aún, incluso si un pedazo de papel, en un caso dado, en verdad sirve como el soporte físico de la deuda en el sentido de la fórmula de Searle, hay muchos otros casos en los cuales las deudas existen sin registro de papel alguno. Searle diría, tal vez, que el soporte físico es proporcionado aquí por marcas (huellas en la memoria, creencias) en los cerebros de las personas; pero una vez más, parece ontológicamente equivocado afirmar que marcas en los cerebros pueden contar como deudas en ciertos contextos. (Y parece equivocado, además, suponer que mediante la destrucción de tales marcas destruiríamos exitosamente a deuda). ${ }^{23}$

Según Smith, la forma lógica «X cuenta como $\mathrm{Y}$ en un contexto $\mathrm{C}$ » no funciona en estos casos porque hay términos ' $\mathrm{Y}$ ' independientes, es decir, no hay un objeto o persona a la que adjudicarle la función de estatus.

Debido a esta crítica, en Making of the Social World Searle modificó su posición original y declaró que puede haber hechos institucionales aún si falta el objeto o la persona a quien adjudicarle la función de estatus, ya que en esos casos el término ' $\mathrm{Y}$ ' funciona como marcador de posición para un conjunto de relaciones de poder reales. Así, la corporación o la sociedad (persona jurídica) es un marcador de posición para relaciones entre entidades que sí poseen existencia física como el directorio, su presidente, el gerente general, etc. Lo mismo puede decirse del dinero electrónico y del ajedrez a la ciega. Siempre hay -dice Searle- marcadores de posición para relaciones de poder en el mundo físico: el propietario del dinero y el poseedor de una torre de ventaja en una partida de ajedrez a la ciega. Por tanto, la función de estatus no implica necesariamente un objeto físico o un soporte material:

Así que no hay objeto o persona que sea la corporación, pero están el presidente, la junta directiva, los accionistas [...] y los poderes deónticos derivados de ellos. Una sociedad es tan solo un marcador de posición para un conjunto de relaciones de poder reales. Lo mismo es válido para el

23 SMITH, Barry \& SEARLE, John: "The Construction of Social Reality: An Exchange". En: American Journal of Economics and Sociology, 62 (1, Special Invited Issue:John Searle's Ideas about Social Reality: Extensions, Criticisms, and Reconstructions), 2003. pp. 285-309 (289). 
dinero electrónico y el ajedrez a la ciega. El propietario del dinero y el poseedor de la reina tienen los poderes relevantes. ${ }^{24}$

Este es un caso interesante de acto ilocucionario, ya que se "crea una función de estatus sin que haya una persona u objeto existente que cuente como el portador de tal función de estatus". ${ }^{25}$ En el ejemplo de Searle, es la ley la que establece los requisitos y condiciones que deben cumplirse para constituir una sociedad anónima. Pero no hay ningún objeto preexistente al que se le adjudique una función de estatus. Ningún objeto del dominio es el portador del nombre "sociedad anónima", pero eso no transforma al término en un predicado vacío, ya que las declaraciones de función de estatus y el seguimiento de reglas constitutivas en un contexto social, dan lugar a la creación de objetos abstractos como las sociedades anónimas. Dicho sea de paso, la creación de una persona jurídica importa una doble declaración: por una parte, del legislador y, por otra, de los socios que constituyen la sociedad como una persona independiente de las personas naturales que la han creado. Se sigue de lo anterior que los socios pueden cambiar, pero la identidad del objeto persona jurídica seguirá siendo la misma.

4.3 Reparos a la noción de intencionalidad colectiva.

Algunos críticos sostienen que la práctica común de asignar estados intencionales a los grupos no es más que una mera ficción. Se trata sencillamente de un recurso metafórico.

Contra la tesis searleana de intencionalidad en sentido fuerte, Raimo Tuomela ${ }^{26}$ defiende que las intenciones no son simples estados mentales igual que las creencias o los deseos, sino que representan compromisos para la acción. Tuomela generaliza el argumento práctico de Von Wright:

1. Nos proponemos causar P.

2. Creemos que no podemos dar lugar a $\mathrm{P}$ a menos que hagamos $\mathrm{Y}$.

3. Por consiguiente, hagamos Y.

Hay un componente normativo que subyace en la intencionalidad colectiva: la aceptación colectiva como deber. Searle no se preocupó explícitamente de la cuestión de la normatividad, que consiste en que al formarse una intención colectiva se crean obligaciones y expectativas entre nosotros. Cuando formamos una intención colectiva

24 SEARLE, John, Making the Social World, op. cit., p. 22, traducción nuestra

25 SEARLE, John, Making the Social World, op. cit., p. 20, traducción nuestra.

26 TUOMELA, Raimo: The Importance of Us: A Philosophical Study of Basic Social Notions. Stanford University Press, 1995. p. 53. 
nos comprometemos con ciertas obligaciones. Searle no reconoce esta normatividad implícita porque para su explicación de las acciones colectivas (i.e. jugar al fútbol) funciona bien apelando a una especie de solipsismo. Pero otros piensan que la normatividad juega un papel esencial en la intencionalidad colectiva. ${ }^{27}$

Otra objeción se relaciona con un problema epistémico. Según Thomasson, debido a que una institución es una creación autoconsciente, el grupo que colectivamente acepta el objeto o hecho institucional no puede ignorar o errar gravemente en cuanto a su naturaleza o contenido. ${ }^{28} \mathrm{La}$ aceptación colectiva del conjunto de condiciones relevantes que han sido declaradas suficientes para la creación de la institución hace que sea el caso de que esas condiciones son suficientes para crear la institución. Dado que el contenido del objeto o hecho institucional es (al menos parcialmente) independiente de las motivaciones de quienes pretenden crearla, aquellos que aceptan colectivamente la institución no pueden errar masivamente sobre la creación de sus normas.

Pero esto nada nos dice sobre la naturaleza de la aceptación colectiva que crea la institución. Simplemente dice que, si hay una institución, entonces hay un cierto grupo cuya aceptación colectiva confiere ese estatus institucional, incluyendo el poder para dar razones y alterar el espacio normativo para quienes están sujetos a la institución. Sin embargo, esta inmunidad epistémica no se extiende a los individuos o grupos que se encuentran fuera del colectivo regido por la institución, lo que deja abierta la posibilidad de la investigación científica y el descubrimiento empírico.

\section{LA TEORÍA DE LA DOCUMENTALIDAD.}

En los últimos años el filósofo italiano Maurizio Ferraris ha contribuido a renovar el debate en la ontología social mediante la publicación de sendos libros y ensayos, en los que expone su doctrina autodenominada "nuevo realismo" (nuovo realismo), teoría de la documentalidad y realismo trascendental. ${ }^{29} \mathrm{La}$ teoría de la documentalidad es tributaria de

27 GILBERT, Margaret, op. cit., 1989.

28 THOMASSON, Amie: "Realism and Human Kinds", op. cit., pp. 588-90.

29 Véase FERRARIS, Maurizio: Manifesto del nuovo realismo, Roma-Bari, Laterza, 2012; FERRARIS, Maurizio: Introduction to New Realism, London, Bloomsbury, 2014; FERRARIS, Maurizio: "Realismo transcendental". En: El giro ontológico, Maurizio Ferraris, Mauricio Beuchot, 1a. ed., Buenos Aires, Círculo Hermenéutico, pp. 13-42. 
tres grandes influencias: (i) la filosofía de la gramática de Jacques Derrida; (ii) el realismo fenomenológico de Adolf Reinach; y (iii) la teoría de los actos de habla de John L. Austin. Por último, se nutre de la perspectiva sociopolítica del economista Hernando de Soto sobre la génesis y naturaleza del capital económico.

El punto de partida de Ferraris es la insuficiencia de las otras teorías explicativas de la realidad social; en particular, la teoría de los hechos institucionales. Una de las objeciones de Ferraris al realismo débil de Searle es que:

o anclamos los objetos sociales en los objetos físicos -y nos encontramos frente al escollo de los objetos sociales amplios, vagos o negativos-, o consideramos que los objetos sociales son "representaciones", y entonces recaemos en posmodernidad, en la imposibilidad de distinguir de iure cien táleros reales de cien táleros ideales ${ }^{30}$.

Paradojalmente, la salida a esta dificultad Ferraris la ve en el pasaje de un antiguo texto de Searle:

A menudo los hechos brutos no se manifestarán como objetos físicos, sino como sonidos que provienen de la boca de la gente o como signos en el papel (o también como pensamientos en su cabeza). ${ }^{31}$

Según Ferraris este pasaje es importante porque los sonidos y los signos no son objetos físicos que se imponen como los Estados o las personas. Poseen menos moléculas que otros objetos físicos, pero esas pocas moléculas son decisivas para configurar el carácter social de ciertos objetos como, por ejemplo, los talonarios bancarios. Las escasas moléculas que se manifiestan como signos distintivos de un talonario bancario son, en realidad, inscripciones. Hay acá una reminiscencia de la filosofía de la escritura de Jacques Derrida y, de hecho, el mismo Ferraris reconoce que su perspectiva intenta reelaborar el textualismo del filósofo francés.

Ferraris reconoce también influencias del economista Hernando de Soto. Como explica Barry Smith la tesis fundamental que de Soto expone en su libro Misterios del Capital, ${ }^{32}$ es que la mayoría de las

30 FERRARIS, Maurizio: ¿Dónde estás? Ontología del teléfono móvil, $1^{\text {a }}$ edición, Marbot Ediciones, Barcelona, España, 2008. p. 276.

31 SEARLE, John: Atti linguistici. Saggio di filosofia del linguaggio, trad. G.R. Cardona, Turín, Boringhieri, 1976. p. 44.

32 DE SOTO, Hernando: El misterio del capital: por qué el capitalismo triunfa en occidente y fracasa en el resto del mundo. Lima, El Comercio, 2000. 
entidades que estructuran nuestra realidad contemporánea son objetos que existen gracias a que hay documentos (en papel o virtuales) que apoyan su existencia. A través de la ejecución de actos documentales (actos de registro, clasificación, transmisión, validación) cambiamos el mundo creando relaciones de propiedad, responsabilidad legal, organizaciones empresariales, y un sinnúmero de redes institucionales invisibles, propias de sociedades altamente sofisticadas.

El rasgo peculiar de estos documentos es su aptitud creadora de acciones, bonos, debentures, pagarés, corporaciones, y hasta grados académicos como magíster o doctor. La lectura que hace Ferraris del libro Misterios del Capital lo lleva a invertir la fórmula searleana (' $\mathrm{X}$ cuenta como $\mathrm{Y}$ en el contexto $C$ ') por ' $C$ constituye $X$ como $Y$ ', donde ' $C$ ' es la inscripción. Parafraseando a Derrida, podría asumirse entonces que en la esfera de los hechos sociales "nada existe fuera del texto". Pero esta asunción -dice Ferraris- resultaría demasiado fuerte en el caso de la ontología social porque implica una pretensión genérica ('C constituye'). De ahí que el autor italiano se incline por un textualismo más débil:

Objeto $=$ Acto Escrito $[\text { Inscrito }]^{33}$

De acuerdo con Ferraris esta fórmula es una condición necesaria, pero no suficiente, para hablar de objetos sociales. Para hablar con propiedad de un objeto social es menester que se dé, en diversas formas, un acto de inscripción, lo que no significa que cualquier acto escrito sea un objeto social (sobre todo un objeto social legítimo), desde que siempre se requiere la existencia de una sociedad cuya génesis no puede ser explicada únicamente a través de la inscripción; y, además, estas sociedades hablan de entidades ambiguas como las "intenciones", que no son sustituibles puramente a través de las inscripciones. Por eso la inscripción debe ser idiomática, en el sentido de que requiere siempre de un registro idiomático, es decir, de un soporte relativo al lenguaje. ${ }^{34}$

\subsection{La noción de huella.}

En una reelaboración de La Gramatología, ${ }^{35}$ Ferraris adopta la noción de huella como sustrato ontológico de su ontología social. La huella que interesa al filósofo italiano es una huella humana: el registro. Dice Ferraris,

33 FERRARIS, Maurizio: ¿Dónde estás? Ontología del teléfono móvil, op. cit., p. 280.

34 Ídem, p. 276.

35 DERRIDA, Jacques: De la gramatología. trad. Oscar del Barco y Conrado Ceretti, Siglo XXI Editores, Ciudad de México, México. 
Con "huella" indico lo que, con un número no muy elevado de moléculas, hace de soporte físico para un registro. Sólo en el caso de los objetos sociales la huella posee un valor constitutivo. En el mundo de los objetos físicos hay huellas sólo para mentes capaces de reconocerlas, por lo tanto, no existen huellas en tanto tales [...] En la constitución de un objeto social las cosas son de otro modo: sin huellas no hay mentes (teorías de la tabula), pero sólo para las mentes hay huellas. ${ }^{36}$

El registro puede ser cualquier forma de inscripción (recuerdos en la mente, signos en un papel, en el computador, en un teléfono móvil, etc.), pero solo cuando se le asigna un valor social adquiere el estatus de inscripción en un sentido estricto. Es el caso de documentos tales como bonos, debentures, acciones, recibos de pago, cheques, pagarés y contratos. Es importante consignar que esta documentalidad no es solo material, sino que también puede ser virtual: tiene tanto valor social una tarjeta de embarque impresa en una hoja de papel como la misma tarjeta de embarque exhibida en la pantalla de un teléfono móvil.

A diferencia de Searle, Ferraris defiende que es la huella y no la intencionalidad colectiva la base central del puente que lleva de los átomos a los parlamentos. El uso de la huella es un debilitamiento, porque debilita tanto la idea de intencionalidad colectiva searleana como el textualismo fuerte postulado por Derrida. Sin embargo, este uso conduce a una paradoja: las sociedades se constituyen sin referencia a las huellas, pero no pueden subsistir sin éstas. Hay una dependencia ontológica de las sociedades respecto de las huellas.

De acuerdo con Ferraris la realidad social no puede explicarse sobre la base de la intencionalidad colectiva porque lo que llamamos "intenciones" son, en rigor, sedimentaciones de huellas. ${ }^{37}$ En esta dirección, si hipotéticamente pudiéramos comunicarnos sólo a través de la telepatía, es muy probable que eso que llamamos "comunicación" no lo sea en lo absoluto.

Para Ferraris lo fundamental no es la comunicación en sí misma, sino la grabación de huellas a través de registros (mapas, archivos, documentos) que constituyen inscripciones. El protagonista de la película Memento (Christopher Nolan, 2000) debido a una inusual patología que afecta su memoria corta, tenía una serie de tatuajes en el cuerpo con frases que

36 FERRARIS, Maurizio, op. cit., p. 283 [Énfasis en el original].

37 Ídem, p. 286. 
le permitían recordar hechos y acciones pretéritas con la finalidad de orientarse en la realidad. En la película, el personaje interpretado por el actor Guy Pearce era capaz de sobrevivir en el caótico mundo real social no por virtud de la comunicación lingüística, sino gracias a las huellas que tenía grabadas en su cuerpo bajo la forma de tatuajes. La mente es una tábula que recoge inscripciones, de ahí que los objetos sociales no son simples trazos en hojas de papel o en soportes virtuales, sino genuinas inscripciones "en" el cerebro.

3.2. La ontología de la teoría de la documentalidad.

En su ensayo Documentalitá. Perché è necessario lasciar tracce, ${ }^{38}$ Ferraris resume la teoría de la documentalidad en once tesis, varias de las cuales tienen que ver con la ontología social. Para empezar, la ontología ferrarisiana es realista (nuovo realismo) y su propósito es dar cuenta del mundo social y de la experiencia cotidiana, esto es, el mundo que está más allá de la realidad científica descrita por las ciencias naturales. El modelo de Ferraris es el catálogo como metáfora del proceso de identificación, clasificación y distinción entre objetos.

Ferraris tiene una pretensión ontológica similar a la que Searle expone en La construcción de la realidad social, ya que intenta explicar la realidad como un todo. Así concebido, el mundo estaría conformado por un conjunto de individuos disímiles: desde rocas, plantas y organismos vivos, hasta contratos, software, bonos y corporaciones. Su ontología considera tres clases de objetos: físicos, ideales y sociales. Los objetos físicos existen en el espacio y en el tiempo, y son concretos y contingentes. Los objetos ideales son abstractos en el sentido de que no son localizables en coordenadas espaciotemporales y dependen de nuestros estados mentales. Por último, los objetos sociales son localizables en el espacio y en el tiempo y, si bien dependen de los sujetos, no por ello son subjetivos.

Como lo expresa Ferraris, "el carácter maravilloso de los objetos sociales, su diferencia, por ejemplo, respecto de los gustos y las imaginaciones, está en el hecho de que, aunque dependan de los sujetos, no son subjetivos". ${ }^{39}$ Los objetos sociales dependen de los sujetos no debido a la intencionalidad (como pensaba Searle), sino en el sentido de que requieren de actos que -

38 FERRARIS, Maurizio: Documentalitá. Perché è necessario lasciar tracce, Roma-Bari, Laterza, 2014.

39 FERRARIS, Maurizio: ¿Dónde estás? Ontología del teléfono móvil, op. cit., p. 184, énfasis en el original. 
como vimos- son inscripciones idiomáticas. Lo que permanece en el objeto social no es el acto, sino la inscripción, la huella y el registro.

En una reformulación del lema derridiano "nada existe fuera del texto", Ferraris postula que "nada social existe fuera del texto". Por extensión, ningún objeto social existe fuera de la inscripción: no basta con la ejecución de actos para producir objetos sociales; es menester que exista, además, un registro. Lo que hace a un matrimonio ser lo que es no es el acto o la intención fenomenológica, sino el registro. Corolario: un objeto no registrado no es un objeto social.

Otra tesis metafísica importante a propósito de los objetos sociales es la nítida distinción entre ontología y epistemología. Esta tesis es relevante para Ferraris porque lo que hay en el mundo no puede ser -por así decirlo"corregido" mediante el pensamiento o las creencias. Pero, como es obvio, nuestro conocimiento del mundo sí puede ser modificado, siendo ésa una de las tareas fundamentales de la epistemología. Dice Ferraris,

El ser, aquello que es la ontología, mientras que aquello que sabemos a propósito de lo que es, la es la epistemología. Es fundamental no confundir estas dimensiones. ${ }^{40}$

Esta distinción no es baladí, pues su confusión ha conducido a lo que Ferraris llama la falacia del ser-saber, que consiste en asumir que gran parte de la realidad (lo que incluye la realidad social) se encuentra construida por nuestros esquemas conceptuales y aparatos perceptivos. Se trata de un error categorial en que cayeron varios posmodernos al eliminar la ontología a favor de la epistemología, reduciendo el mundo a "una fábula y, por tanto, un producto discursivo, textual; en su máxima expresión, una especie de textualismo fuerte". ${ }^{41}$

\subsection{Crítica de la teoría de la documentalidad.}

La ontología social defendida por Ferraris es todavía demasiado reciente como para que tenga lugar un balance definitivo. Sin embargo, parece plausible la formulación de los siguientes problemas:

40 FERRARIS, Maurizio: "Reconstruir la deconstrucción". En: II Jornadas Internacionales de hermenéutica: La hermenéutica en diálogo con las ciencias humanas y sociales: convergencias, contraposiciones y tensiones, (trad. del italiano por María José Rossi), evento celebrado entre el 6 y el 8 de julio de 2011 en Buenos Aires, Argentina.

41 JEREZ, José Luis: “¿Por qué se habla nuevamente de realismo?”, disponible en la página web https://nuovorealismo.files.wordpress.com/2013/12/por-quecc81-se-hablanuevamente-de-realismo.pdf 
(i) En primer término, si bien hay algo de verdad en la idea de que debemos tener en cuenta el cerebro (la mente como tábula) y los estados mentales de los individuos que producen actos sociales, no queda claro cómo es que las huellas pueden crear, por ejemplo, obligaciones "reales", en lugar de meros sentimientos o creencias. Dicho de otro modo, no es evidente el procedimiento a través del cual los sujetos producen objetos sociales. ¿Es una combinación entre el acto y el objeto físico?

(ii) En segundo lugar, si lo que permanece en el objeto social no es el acto (i.e. la promesa) sino la inscripción, entonces ¿bajo qué condiciones se extingue, por ejemplo, una obligación? Una vez creado el objeto social ¿depende de nosotros los humanos? Y si existen relaciones de dependencia, ¿cuáles son?

(iii) Por último, en los últimos años ha surgido un enfoque similar al realismo defendido por Ferraris, que rescata la hermenéutica como un elemento fundamental para la comprensión de la realidad social. Sus defensores -Mauricio Beuchot y José Luis Jerez- lo han denominado nuevo realismo analógico ${ }^{42}$. Está por verse si esta perspectiva que pone énfasis en la tradición hermenéutica de Heidegger, Gadamer y Ricoeur, es una mejor explicación de los problemas filosóficos que plantean los objetos sociales, pero no podemos extendernos en su consideración.

\section{CONCLUSIONES.}

Pese a ser una propuesta interesante y robusta sobre cómo se construye la realidad social, la teoría de los hechos institucionales de John Searle ha sido cuestionada en prácticamente todos sus planteamientos básicos, y las mejoras que el filósofo norteamericano introdujo en su libro más reciente -Making the Social World: The Structure of Human Civilization-, si bien son interesantes y resuelven varias de las críticas, no logran superar los problemas de autorreferencialidad de ciertos conceptos opacos, ligados a varias disciplinas, pero especialmente al ámbito de la ciencia económica, tales como deflación, inflación, recesión, etc. Por otro lado, coincidimos con Brian Epstein en que la teoría searleana otorga un énfasis excesivo (y no del todo justificado) a la noción de intencionalidad, a tal punto que podría prescindirse de él y, sin embargo, mantener una teoría sólida respecto de los hechos y objetos sociales. De hecho, esta es una de las tesis de Maurizio

42 Véase BEUCHOT, Mauricio y JERÉZ, José Luis: Manifiesto del nuevo realismo analógico. Neuquén Ed. Círculo Hermenéutico, 2013. 
Ferraris con su teoría de la documentalidad. Ahora bien, resultaría posible defender una tesis débil sobre la intencionalidad (individual y colectiva) en la ontología social, pero entonces el rol explicativo de la teoría de Searle se reduciría ostensiblemente, comprometiéndose el postulado esencial de que la ontología social, en tanto porción de una única realidad, es la base de las ciencias sociales y de la filosofía política.

En cuanto a la teoría de la documentalidad de Maurizio Ferraris, es cierto que la mayoría de los actos de habla son, de hecho, actos inscritos, ya que sin la existencia de registros no hay forma de que los actos performativos puedan engendrar objetos sociales complejos como matrimonios, sinfonías, leyes o constituciones políticas. En su reelaboración del lema subversivo de Derrida - "nada hay fuera del texto"- Ferraris se ve obligado a admitir que existen hechos y objetos precisamente fuera del texto, tales como las ciencias duras (física, química, biología) cuya existencia, además, es independiente de cualquier registro, inscripción o huella. De hecho, el mundo físico, el de la química orgánica y el de la biología evolucionista existieron millones de años antes de los seres humanos y, por consiguiente, antes de imaginar siquiera la idea de huellas, registros e inscripciones innatas en el cerebro del homo sapiens.

Ahora bien, si Ferraris y de Soto tienen razón, el lema derridiano reformulado sería "nada social existe fuera del texto" y, en este sentido, su explicación de la ontología social resulta muy interesante. Por lo general, no solemos prestar atención a objetos sociales como los cheques, bonos, matrimonios, constituciones políticas y softwares, ni tampoco a hechos económicos como la inflación, deflación o recesión, sino sólo cuando ellos no funcionan o lo hacen defectuosamente.

Sin embargo, quedan en pie una serie de objeciones como las esbozadas supra, donde la más importante parece ser la explicación de Ferraris respecto de la subsistencia o permanencia de los objetos sociales en el tiempo. La críptica formulación objeto = acto inscrito es dudosa, incluso si se reconoce que los hechos sociales están sostenidos por inscripciones, como postula el filósofo italiano. En efecto, pese a la importante función que Ferraris asigna al cerebro y a los estados mentales en tanto "captadores innatos de huellas", no queda claro cómo es que las huellas pueden crear, por ejemplo, obligaciones "reales", en lugar de meros sentimientos o creencias. Dicho de otro modo, no es evidente el procedimiento a través del cual los sujetos producen objetos sociales. Como nos preguntamos antes, ¿es una combinación entre el acto y el objeto físico? 
Desde el punto de vista ontológico, cabe preguntar -como lo hace Amie Thomasson- ¿dónde "está" la Constitución de los Estados Unidos? $\mathrm{Si}$ asumimos la propuesta de Ferraris, habría que concluir que la Carta Fundamental está en el texto original firmado por los padres fundadores y, mejor aún, en todas las copias impresas y digitales de la Constitución e, incluso, en la mente de los hablantes. Sin embargo, esto parece contraintuitivo: la constitución política no parece residir en la cabeza de los ciudadanos de Estados Unidos, y tampoco en las copias e impresiones del texto original. A esta objeción Ferraris responde que el hecho u objeto social se "caracteriza" por estar registrado en un papel o en un soporte material o digital e "inscrito" en el cerebro de los ciudadanos, pero esta explicación reduce la potencialidad de la teoría a sólo uno de los actos de habla de la taxonomía de J. L. Austin: aquellos que están inscritos en la cabeza de los hablantes.

Por último, si lo que permanece en el objeto social no es el acto (i.e. la promesa) sino la inscripción, entonces ¿bajo qué condiciones se extingue, por ejemplo, una obligación? Una vez creado el objeto social, ¿depende de nosotros los humanos? Y si existen relaciones de dependencia, ¿cuáles son?

Todos estos son flancos abiertos para la teoría de Ferraris y de su elucidación depende el éxito de su teoría de la documentalidad.

\section{BIBLIOGRAFÍA.}

ANSCOMBE, Elizabeth: "Ethics, Religion and Politics". En: The Collected Philosophical Papers of G.E.M. Anscombe. Oxford: Basil Blackwell, vol. 3, 1981.

BENGOETXEA, Joxerramon: "Teoría institucional del derecho". En: Enciclopedia de Filosofía y Teoría del Derecho, vol. uno, J. Fabra y A. Núñez ed., UNAM, México, 2015.

BEUCHOT, Mauricio y JEREZ, José Luis: Manifiesto del nuevo realismo analógico. Neuquén Ed. Círculo Hermenéutico, 2013.

BUENO, Gustavo: Ensayos Materialistas, Taurus, Madrid., 1972

BURMAN ASA: "Extending the scope of Searle's Theory of Social Reality - How to account for opaque social phenomena like power structures"; ProtoSociology, 2015. 
DE SOTO, Hernando: El misterio del capital: por qué el capitalismo triunfa en occidente y fracasa en el resto del mundo. Lima, El Comercio, 2000.

DERRIDA, Jacques: De la gramatología, trad. Oscar del Barco y Conrado Ceretti, Siglo XXI Editores, Ciudad de México, México, 1986.

EPSTEIN, Brian: "Social Objects Without Intentions". Anita Konzelmann Ziv \& Hans Bernhard Schmid (eds.), Institutions, Emotions, and Group Agents: Contributions to Social Ontology, 2013.

FERRARIS, Maurizio: ¿Dónde estás? Ontología del teléfono móvil. $1^{\mathrm{a}}$ edición, Marbot Ediciones, Barcelona, 2008.

FERRARIS, Maurizio: Documentality, New York: Fordham University Press, 2009.

FERRARIS, Maurizio: "Reconstruir la deconstrucción", II Jornadas Internacionales de hermenéutica: La hermenéutica en diálogo con las ciencias humanas y sociales: convergencias, contraposiciones y tensiones, (trad. del italiano por María José Rossi), Buenos Aires, 2011.

FERRARIS, Maurizio: Manifesto del nuovo realismo, Roma-Bari: Laterza, 2012.

FERRARIS, Maurizio: Introduction to New Realism, London, Bloomsbury, 2014.

FERRARIS, Maurizio: Documentalitá. Perché è necessario lasciar tracce, Roma-Bari, Laterza, 2014.

FERRARIS, Maurizio: "Realismo transcendental". En: El giro ontológico. Maurizio Ferraris; Mauricio Beuchot, 1a. ed. Buenos Aires, Círculo Hermenéutico, 2015.

GILBERT, Margaret: On Social Facts, Routledge, 1989.

HUSSERL, Edmund: Ideen zu einer reinen Phánomenologie und phánomenologischen Philosophie. Drittes Buch. Die Phánomenologie und die Fundamente der Wissenschaften (Ideen III), Beilagen, §6, Hua V, Den Haag, 1952.

JEREZ, José Luis: “¿Por qué se habla nuevamente de realismo?”, disponible en la página web https://nuovorealismo.files.wordpress.com/2013/12/porquecc81-se-habla-nuevamente-de-realismo.pdf 
JIMENEZ CANO, Rosa: "El derecho como institución social: Searle y Hart". En: Entre la ética, la política y el derecho: estudios en homenaje al profesor Gregorio Peces-Barba, tomo II: Teoría y Metodología del Derecho, Madrid, Dykinson, 2008.

LUCENA, Isabel: "La intencionalidad colectiva: un modelo explicativo de la acción y de la aceptación social”. En: Revista Telemática de Filosofía del Derecho, N¹4, 2011.

MAÑALICH, Juan Pablo: "Norma e imputación como categorías del hecho punible". En: Revista de Estudios de la Justicia, N¹2, 2010.

PUTNAM, Hilary: "The meaning of "meaning"'. En: Philosophical Papers, Vol. 2: Mind, Language and Reality. Cambridge University Press, 1975.

SEARLE, John: Atti linguistici. Saggio di filosofia del linguaggio, trad. G.R. Cardona, Turín: Boringhieri, 1976.

SEARLE, John: "Collective Intentions and Actions". En: Cohen, P. Morgan, J y Pollack, M. E. (eds): Intentions in Communications. Cambridge, MA: Bradford Books, MIT Press, 1990.

SEARLE, John: La construcción de la realidad social, Barcelona, Paidós, 1997.

SEARLE, John: Making the Social World: The Structure of Human Civilization, New York, Oxford University Press, 2010.

SMITH, Barry: “John Searle: From speech acts to social reality”. En: B. Smith (ed.), John Searle. Cambridge, Cambridge University Press, 2003.

THOMASSON, Amie: "Foundations for a Social Ontology". En: Protosociology: An International Journal of Interdisciplinary Research, vol. 18-19, 2003.

THOMASSON, Amie: "Realism and Human Kinds". En: Philosophy and Phenomenological Research, 67, 2003.

TUOMELA, Raimo: The Importance of Us: A Philosophical Study of Basic Social Notions. Stanford University Press, 1995.

TUZET, Giovanni: Ontología social y práctica jurídica: un debate, tr. D. Dei Vecchi. Discusiones, XIV, 2014. 